\title{
Clinical Insights for Gastroenterology and Hepatology Providers during the COVID-19 Pandemic
}

\author{
Cristian Gheorghe
}

President of Romanian Society

of Gastroenterology and

Hepatology (SRGH)

On behalf of SRGH and

affiliated societies

Address for correspondence:

Cristian Gheorghe

Fundeni Clinical Institute

Center of Gastroenterology

and Hepatology,

Carol Davila University of

Medicine and Pharmacy

Fundeni Street, 258

Bucharest, Romania

drgheorghe@xnet.ro
Received: 21.05.2020

Accepted: 28.05.2020
Coronavirus disease 2019 (COVID-19) is a disease caused by severe acute respiratory syndrome coronavirus 2 (SARSCoV-2), first detected in China in December 2019. In January 2020, WHO decided to define the outbreak of COVID-19 as a public health emergency of international concern and then, on March 11, WHO declared it to be a pandemic with more than 300,000 cases worldwide reported as of March 2020, worldwide, excluding Antarctica, spreading on a logarithmic scale in Europe. Although most patients infected with SARS-CoV-2 experienced a mild illness, about $5 \%$ of patients developed severe lung injury or even multi-organ dysfunction, resulting in a $1-4 \%$ case fatality ratio [1]. As of May 13, 2020, more than 4.1 million confirmed cases of COVID-19 have been reported, with more than 280,000 attributable deaths around the world [2]. The first patient with COVID-19 in Romania was confirmed on February 26, 2020 in the southern part of the country, with the disease spreading rapidly thereafter throughout the country. Subsequently, strict social distancing policies were implemented by the Romanian government as of March 15, 2020, to mitigate the spread of COVID-19. Until May 22nd, 17,712 cases of SARS-CoV-2 infection with 1,159 deaths/ fatalities had been reported in our country.
Key symptoms of COVID-19 include cough and fever, but a significant number of patients can exhibit gastrointestinal (GI) symptoms, including diarrhea $[3,4]$.

The main route of virus transmission is via aerosolized droplets from the pharynx and bronchioles of infected individuals (cough-generated aerosol clouds can spread/extend 1-2 meters from the patient) [5]; the possibility of fecal-oral transmission also exists [6]. SARS-CoV-2 virus particles can be deposited on surfaces where they may remain detectable for up to 72 hours [7]. More than $40 \%$ of all SARS-CoV-2 infections may be transmitted prior to the index case becoming symptomatic; infected individuals are infectious/contagious as early as 24 hours prior to symptom onset till at least 5-6 days thereafter [8].

SARS-CoV-2 enters cells via the angiotensin-converting enzyme 2 (ACE2) receptor. ACE2 receptors are expressed on different cell types in the body and appear to be most expressed in the intestine but can be found in many other organs including lungs, heart, kidney, liver and pancreas [9]. ACE2 receptors are also expressed by endothelial cells and cardiovascular complications are rapidly emerging as a key threat of COVID 2019, in addition to respiratory disease. We do not yet know if direct virus-induced tissue damage, systemic cytokine storm, or the synergistic effects of both contributes to the multiple organ dysfunction of severe COVID-19 patients [10].

We do not know yet the extension, duration and protection conferred by acquired immunity to SARS-CoV-2.

In my opinion, COVID-19 is a systemic disease, showing respiratory and cardiovascular involvement.

We know that the most common symptoms of COVID-19 are fever, fatigue and respiratory symptoms (cough, dyspnea), but, as gastroenterologists, we have to highlight that a significant proportion of patients will have alterations of bowel habits or other digestive symptoms (nausea, vomiting, abdominal pain) [4]. Digestive symptoms may reflect the inoculation of the GI tract from swallowing SARS-CoV-2 and linking to ACE2 intestinal receptors [11]. Recent reports have focused on both the GI-related manifestations of COVID-19, as well as the fact that virus is detectable in the stool long after the resolution of respiratory symptoms [12]. Extra-pulmonary symptoms occur early in the disease course. Gastrointestinal symptoms, including anorexia, nausea, vomiting, abdominal pain, and/or diarrhea may occur early, but are rarely the sole 
presenting feature; GI symptoms may be associated with poor clinical outcomes including a higher risk of mortality [12]. While symptoms remain mild throughout the course of infection in the majority of patients, older patients were identified to be at higher risk of a fatal disease course, as well as patients with co-morbidities such as arterial hypertension, diabetes and coronary heart disease.

It is important to keep in mind that digestive symptoms are commonly encountered in the community and in most instances, new-onset diarrhea, nausea, or vomiting are not associated with COVID-19. Nonetheless, clinicians should recognize that new-onset acute digestive symptoms in a patient with possible COVID-19 contact, should at least prompt consider the illness, particularly during the times of high COVID-19 incidence and pandemic prevalence [13]. Failure to recognize these patients early in the disease course, may often lead to unwitting spread of the disease among outpatients with mild illness who remain undiagnosed and unaware of their potential to infect others.

The comorbidity spectrum of digestive conditions and its impact on the course of COVID-19 remains largely unknown, as is the effect of COVID-19 on specific therapy and outcome of various GI and liver diseases. So far, we have limited data on patients with inflammatory bowel diseases (IBD), liver diseases, and digestive cancers during the COVID-19 pandemic.

Patients with IBD do not appear to be at an increased baseline risk for infection with SARS-CoV-2 or development of severe COVID 19. According to current evidence and expert recommendations, during the SARS-CoV-2 pandemic, on-going medication for stable IBD should be continued in order to maintain remission and avoid IBD flares, complications and the need for surgery, but clinicians need to halt immunosuppressive therapies and biologics when there is a risk of COVID-19 infection [14].

Patients with cancers are at higher risk for infection, serious illness, admission to the ICU and death from COVID-19. This risk is related to the immunosuppressive status caused by the disease itself, malnutrition, and the effect of treatment (surgery, chemo- and radiotherapy). The risk of mortality from a long-lasting postponement of interventions such as surgery, chemotherapy infusions, or scheduled radiotherapy may become higher than that of a serious hospital-dependent COVID-19 disease. Therefore, medical treatment for patients with GI/liver cancer cannot be discontinued, and specific infection prevention measures should be applied to these immunocompromised patients if they have to visit hospital facilities for therapy and evaluation of their response to therapy [15].

Patients with autoimmune liver disease, advanced liver disease and those after liver transplantation are increased risk of infection and/or a severe course of COVID-19 due to disease-associated immune dysfunction. On the other hand, systemic SARS-CoV-2 infection is often associated with elevated alanine aminotransferase levels, reduced platelet counts and reduced albumin levels at the time of admission. In addition, the current pandemic requires unusual allocation of healthcare resources which may negatively impact the care of patients with chronic liver disease that continue to require medical attention (surveillance for varices and hepatocellular carcinoma, management of hepatocellular carcinoma, temporarily suspended transplant activities etc.) [16].

All endoscopic procedures should be considered aerosolgenerating procedures and given this high rate of infection transmission from asymptomatic individuals, all patients undergoing GI endoscopy need to be considered "high risk patients". Accordingly, gastroenterological societies around the world have recommended the suspension of screening, surveillance and non-urgent endoscopy, but we have to be prepared to return to routine endoscopy in the next 6 months after the COVID-19 pandemic. We need to test all the patients scheduled for endoscopy; personal protective equipment including gowns, gloves, hair covers, and eye protection (goggles or face shields) have to be worn in order to minimize hazards that cause workplace injuries, especially considering the fact that contamination can occur through the nose, eyes and mouth [17].

Since the WHO declared COVID-19 a global pandemic on March 2020 Romanian Medical Scientific Societies have started implementing infection control measures, planning for a surge capacity in health-care facilities, and proposing a triage of health-care services. Hospital leadership and individual providers are facing increasingly difficult decisions about how to conserve critical resources, such as hospital and intensive care unit beds, respirators, transfusion capacity as well as protective gear that is vital for protecting patients and staff from unnecessary exposure and intra-hospital transmission. The Romanian Society for Gastroenterology and Hepatology (SRGH), the Romanian Society for Digestive Endoscopy (SRED) and the Romanian Society for Minimal Invasive Surgery (ARCE) recommended suspension of all elective procedures (endoscopic or surgical) and rescheduling in order to mitigate the COVID-19 spread and preserve resources. The websites of our national societies involved in the management and counselling of patients with digestive conditions have specified what gastrointestinal procedures should be considered urgent, semi-urgent and elective and how to stay safe performing these procedures (www.srgh.ro, www.sred.ro, www.rccc.ro, www.roald.ro, www.aspiir.ro, www. arce.ro). However, this has raised difficult questions about which procedures can be safely postponed and for how long. For example, while there is data to support a delay of up to 3-6 months for patients undergoing colonoscopy for positive fecal immunochemistry test and this is likely generalizable to patients undergoing colonoscopy for polyp surveillance, the data to support delays for procedures such as endoscopic mucosal resection for large polyps, are lacking. Moreover, there may be added issues around patient anxiety or worry and concerns about medico-legal risks that may influence decisions about procedures.

For the present and the immediate future (likely the next one or two years or, possibly, yearly - we still do not know), there are many goals to achieve in order to win the war against this deadly pandemic virus. Until a stable COVID-19 vaccine will be available and accessible, a prospect which is not guaranteed, the way out of this crisis will be built on the established public health practices of testing, contact tracing, quarantine of contacts and isolation of cases. Aside from these, we should stay well informed about COVID-19, protect our healthcare workers, reshape and properly equip our hospitals 
and other medical facilities for both routine service and future crises and expand appropriate services to internet platforms.

Conflicts of interest: None to declare.

\section{REFERENCES}

1. Guan WJ, Ni ZY, Hu Y, et al. Clinical characteristics of coronavirus disease 2019 in China. N Engl J Med 2020;382:1708-1720. doi:10.1056/ NEJMoa2002032

2. World Health Organization. Coronavirus disease 2019 (COVID-19) situation report - 114. 13 May 2020. Available at:https://www.who. int/emergencies/diseases/novel-coronavirus-2019/situation-reports. Accessed May 14, 2020.

3. Huang C, Wang, Y, Li X, et al. Clinical features of patients infected with 2019 novel coronavirus in Wuhan, China. Lancet 2020;395:497-506. doi:10.1016/S0140-6736(20)30183-5

4. Gu J, Han B, Wang J. COVID-19: Gastrointestinal manifestations and potential fecal-oral transmission. Gastroenterology 2020;158:15181519. doi:10.1053/j.gastro.2020.02.054

5. Bouroulba L, Dehandschoewercker E, Bush JWM. Violent expiratory events: on coughing and sneezing. J Fluid Mech 2014;745:537-563. doi:10.1017/jfm.2014.88

6. Wu D, Wu T, Liu Q, Yang Z. The SARS-CoV-2 outbreak: what we know. Int J Infect Dis 2020;94:44-48. doi:10.1016/j.ijid.2020.03.004

7. van Doremalen N, Bushmaker T, Morris DH, et al. Aerosol and surface stability of SARS-CoV-2 as compared with SARS-CoV-1. N Engl J Med 2020;382:1564-1567. doi:10.1056/NEJMc2004973

8. Ferguson NM, Laydon D, Nedjati-Gilani G, et al. Report 9: Impact of non-pharmaceutical interventions (NPIs) to reduce COVID-19 mortality and healthcare demand. Imperial College London, UK. 2020 March 16. doi:10.25561/77482
9. $\mathrm{Xu} \mathrm{H}$, Zhong L, Deng J, et al. High expression of ACE2 receptor of 2019-nCoV on the epithelial cells of oral mucosa. Int Oral Sci2020;12:8. doi:10.1038/s41368-020-0074-X

10. Li H, Liu L, Zhang D, et al. SARS-CoV-2 and viral sepsis: observations and hypotheses. Lancet 2020:395;1517-1520. doi:10.1016/S01406736(20)30920-X

11. Pan L, Mu M, Yang P, et al. Clinical characteristics of COVID-19 patients with digestive symptoms in Hubei, China: a descriptive, cross-sectional, multicenter study. Am J Gastroenterol 2020;115:766-773. doi:10.14309/ ajg.0000000000000620

12. Wu Y, Guo C, Tang L, et al. Prolonged presence of SARS-CoV-2 viral RNA in faecal samples. Lancet Gastroenterol Hepatol 2020;5:434-435. doi:10.1016/S2468-1253(20)30083-2

13. Mao R, Qiu Y, He JS, et al. Manifestations and prognosis of gastrointestinal and liver involvement in patients with COVID-19: a systematic review and meta-analysis. Lancet Gastroenterol Hepatol 2020 May 12. doi:10.1016/S2468-1253(20)30126-6

14. Kennedy NA, Jones GR, Lamb CA, et al. British Society of Gastroenterology guidance for management of inflammatory bowel disease during the COVID-19 pandemic. Gut 2020;69:984-990. doi:10.1136/gutjnl-2020-321244

15. Mauri D, Kamposioras K, Tolia M, et al. Summary of international recommandations in 23 languages for patients with cancer during the COVID-19 pandemic. Lancet Oncol 2020 May 13. doi:10.1016/S14702045(20)30278-3

16. Boetler T, Newsome PN, Mondelli MU, et al. Care of patients with liver disease during the COVID-19 pandemic: EASLESCMID position paper. JHEP Rep 2020;2:100113. doi:10.1016/j. jhepr.2020.100113

17. Gralnek IM, Hassan C, Beilenhoff U, et al. ESGE and ESGENA position statement on gastrointestinal endoscopy and the COVID-19 pandemic. Endoscopy 2020;52:483-490. doi:10.1055/a-1155-6229 